Cahiers de recherches médiévales

\title{
Une épopée à la mode humaniste
}

Les Visions d'Oger le Dannoys au Royaulme de Fairie

\section{Emmanuelle Hoyer-Poulain}

\section{(2) OpenEdition \\ Journals}

Édition électronique

URL : https://journals.openedition.org/crm/2476

DOI : $10.4000 / \mathrm{crm} .2476$

ISSN : 1955-2424

Éditeur

Honoré Champion

Édition imprimée

Date de publication : 12 décembre 1996

Pagination : 12-23

ISSN : 1272-9752

Référence électronique

Emmanuelle Hoyer-Poulain, «Une épopée à la mode humaniste », Cahiers de recherches médiévales [En ligne], 2 | 1996, mis en ligne le 24 juin 2008, consulté le 15 décembre 2022. URL : http:// journals.openedition.org/crm/2476; DOI : https://doi.org/10.4000/crm.2476 


\section{Une épopée à la mode humaniste : Les Visions d'Oger le Dannoys au Royaulme de Fairie.}

"Particulièrement curieuses": c'est l'intrigante définition que donne en 1922 Henri Franchet' des Visions d'Oger le Dannoys au royaulme de Fairie, rédigées en 1542 par François Habert. De fait ce texte ${ }^{2}$ marque une étrange étape dans l'histoire de la légende d'Ogier. A coup sûr inspiré par la mise en prose de la version en alexandrins, largement répandue au XV' siècle, l'ouvrage se révèle difficilement classable à plus d'un titre.

Dans la bibliographie de son auteur tout d'abord: François Habert, poète mineur (quoique reconnu de son temps, puisqu' officiellement « poète du roi ») de la cour d'Henri II, s'est essentiellement illustré dans des traductions (Ovide, Horace, Caton), de nombreuses épîtres de circonstance, ainsi qu'un Philosophe parfait, un Temple de Vertu, et quelques poèmes à tonalité mythologique. D'inspiration médiévale, point, si ce n'est dans ces «curieuses" Visions d'Oger" ${ }^{3}$.

De même, François Habert s'est bien peu livré au genre des Visions ${ }^{4}$, et s'il s'y adonne ici, c'est sur un mode fort personnel. Il faut en effet rappeler que, dès le Moyen Age, les $V_{i}$ siones s'inscrivent dans une perspective hagiographique et eschatologique. Un personnage, (généralement un religieux) gravement malade le plus souvent, se trouve transporté par l'esprit au Ciel, en Enfer ou encore au Purgatoire. Cette vision de l'au-delà, qui se clôt par un douloureux retour dans l'enveloppe corporelle, se veut pleine d'enseignement, pour le principal protagoniste de l'aventure comme pour le public, incité à la pénitence et à la vertu. Or on verra que F. Habert, s'il assigne globalement une fonction similaire à son ouvrage, exploite cependant un matériau si différent qu'on ne saurait lui accorder tout à fait la même signification qu'aux Visiones traditionnelles.

C'est précisément ce matériau qui constitue l'objet de notre étude. Tel qu'il est, notre texte donne en effet à lire une certaine adaptation d'une œuvre médiévale à la Renaissance. En déterminant ce qui a été conservé, transformé ou ajouté, nous tenterons de comprendre l'intérêt que pouvait représenter la reprise «humaniste» d'un texte issu de la tradition de la chanson de geste.

\footnotetext{
${ }^{1}$ Henri Franchet est le seul «contemporain » qui ait mené une rapide étude d'ensemble de l'œuvre de François Habert : voir H. Franchet, Le Philosophe Parfaict et le Temple de Vertu de Frangois Habert, Paris, Champion, 1922. Voir aussi l'introduction de J. Lewis au Songe de Pantagruel, publié dans Etudes Rabelaisiennes, tome XVIII, Genève, Droz, 1985, pp. 103-162.

${ }^{2}$ Quarante-huit pages non numérotées (environ 1550 vers), réparties en trois livres, et précédées d'un dizain à la duchesse de Touteville. Visions d'Oger le Dannoys au Royaulme de Fairie, Paris, Ponce Roffet dict le Faulcheur, 1542 (BN, Res. Ye 1597)

${ }^{3}$ Plusieurs allusions dispersées dans l'ensemble de l'cuvre montrent cependant que l'auteur connait bien la littérature médiévale, notamment la Chanson de Roland et le Roman de la Rose, mais également toutes les proses épiques ou arthuriennes (voir Le Songe de Pantagruel, ed. citée, p. 146). Nous étudierons plus loin les nombreuses références qui émaillent le texte qui nous occupe.

${ }^{4}$ Un seul autre ouvrage de même nature dans la bibliographie de F. Habert : Le Livre des Visions Fantastiques, allégorie d'inspiration mythologique. Quant au Songe de Pantagruel, il relève plutôt du genre de l'oraculum : Gargantua y apparait en songe à son fils et le conseille par le biais d'une allégorie pastorale.
} 


\section{L'inspiration médiévale.}

La nature des épisodes réécrits ne laisse aucun doute sur le texte-source: il ne peut s'agir que d'une mise en prose ${ }^{5}$ de la version en alexandrins (vers 1335) de ce qui est devenu le Roman d'Ogier le Danois ${ }^{6}$. Rappelons qu'au début du XIV siècle la Chevalerie Ogier s'est trouvée notablement allongée, par une suite aux Croisades d'abord, et par une suite féerique ensuite.

Le texte de François Habert opère quant à lui un découpage précis dans le roman qu'il utilise. Seul lui importe l'épisode en Féerie (propre aux remaniements), dont il reprend à grands traits les principales étapes: l'accostage forcé d'Ogier sur la pierre d'Aimant, l'intervention de la fée Morgue, depuis longtemps éprise du chevalier, la couronne d'oubli et l'anneau d'éternelle jeunesse qu'elle lui offre, les combats d'Ogier, contre les Luytons qui assiègent le château de Morgue, mais aussi contre un serpent gardien du jardin merveilleux, l'accueil musical des demoiselles-fées, et enfin l'union avec Morgue et la naissance d'un fils. Habert ne retient donc de la suite féerique qu'une étape assez brève, exactement limitée au séjour d'Ogier auprès de Morgue : le retour d'Ogier en France et sa disparition finale ne sont pas pris en compte dans le poème du Xvie siècle.

Outre la reprise de ces grands épisodes, le lecteur trouve aisément la reproduction de détails qui témoignent d'une lecture attentive de l'œuvre initiale.

Ainsi, le récit de la naissance du fils d'Ogier et de Morgue transpose et adapte celui de la naissance difficile du héros : dans le roman, Ogier

devint si grant et si gros [ dans le ventre de sa mère] que chacun disoit qu'elle feroit deux enfans [...] et quant vint au terme que la dame deut enfanter elle fut tant malade et eut tant de mal que après qu'elle fut délivré[e] il convint qu'elle prist mort (pp. 5-6).

Dans nos Visions,

D'ung bel enfant son amye accoucha :

Mais ce ne fut sans endurer douleur

A l'enfanter, pour l'estrange grandeur

De cest enfant, qui adoncques fut veu,

Sur tous humains de grand beaulté pourveu (troisième livre).

De même, certaines descriptions se souviennent de leur modèle : chacune des apparitions de Morgue dans le roman d'Ogier évoque la blancheur de son vêtement et l'admiration qu'elle suscite. C'est

\footnotetext{
${ }^{5}$ Toutes nos citations proviennent de l'incunable de Vérard, version la plus ancienne (1498) que nous possédions, dont le fac-similé a été édité par K. Togeby : Ogier le Dannoys, Roman en prase du $x \boldsymbol{f}$ siècle, Munsksgaard, 1967.

${ }^{6}$ Le remaniement en décasyllabes, plus ancien (vers 1310) n'a donné lieu à aucune reprise et n'offrait donc aucune édition. Par ailleurs, l'entrée en Féerie ainsi que la description de la vie en Avalon diffèrent assez nettement dans les deux remaniements pour qu'aucun doute ne subsiste sur l'origine du texte de Habert.
} 
une moult belle dame toute vestue de blanc si bien et si richement aomée que c'estoit ung grant triumphe que de la veoir (p. 270), une tant belle dame habillée d'ung damas blanc portant avec soy une grande lumière [...] et pourquoy tous ceulx qui la estoient s'en esmerveillerent grandement. (p. 312).

Or, dans l'unique portrait qu'il nous livre de Morgue, Habert écrit:

Elle exoma son corps d'un blanc samis,

Le plus exquis qui onc fut apperceu

Par elle mesme en ouvraige tyssu.

Ses blonds cheveulx jusqu'en terre espandus

Maintz chevaliers esbahys ont rendus,

Sa grand beauté qui toutes aultres retarde

Rend esbahy celuy qui la regarde (premier livre).

Cette reprise d'ensemble, et parfois de détail, ne doit cependant pas leurrer : le roman d'Ogier fournit une trame narrative que $F$. Habert remanie à sa guise.

Ainsi, l'accueil du héros à Avalon fournit un bon exemple de manipulation du texte originel : dans le roman en prose, Ogier rencontre d'abord le cheval-faé Papillon, qui le sert à table en multipliant les prodiges. Après une première nuit passée seul dans le château d'Aymant, le héros doit combattre un «serpent » (comme ensuite chez Habert, il s'agit d'un dragon) qui l'empêche de sortir: Ogier tue finalement l'animal et suit « une petite sente qui le mena en un vergier tant bel et tant plaisant que c'estoit un petit paradis à veoir » (p. 270). Choisissant (on est tenté de dire naturellement) de goûter une pomme, Ogier se trouve plongé dans une grande faiblesse, dont Morgue, apparaissant alors, le tire en lui passant l'anneau de jeunesse. On voit que cet épisode, très touffu et riche en éléments merveilleux, correspond à la traditionnelle mise à l'épreuve du héros (qui va jusqu'à une mort symbolique) à l'entrée de l'Autre Monde.

L'œuvre du XvI siècle, quant à elle, retient bien le thème de l'épreuve, mais en lui accordant une signification sur laquelle nous reviendrons: le combat intervient désormais après le mariage avec Morgue, et justifie le droit d'Ogier à régner. Le voyant vainqueur, la fée lui expose en effet

Que c'estoit luy qui estoit Empereur?

De ce pays, et le vray conqueteur (fin du premier livre).

D'autre part, Habert élague sérieusement le merveilleux, en se limitant aux éléments chrétiens, et en inversant la valeur de la pomme, (la mort apparente constituait ici une trace du merveilleux celtique) qui devient récompense cueillie pour le héros «dans le jardin d'immortelle plaisance " (à l'imaginaire chrétien se superpose ici la référence mythologique). Il s'agit donc bien d'une réécriture, dont nous trouverons trace jusque dans les passages directement issus du modèle.

\footnotetext{
${ }^{7}$ Ce terme d'empereur ne manque pas d'intérêt pour la dimension politique du texte : c'est en effet seulement depuis François I que le roi de France se donne pour empereur de son royaume, et ce pour des raisons de politique extérieure.
} 
Reste que ce premier examen du texte semble indiquer clairement que l'aspect épique, le plus ancien dans l'œuvre, n'a pas retenu l'attention de Habert. La partie du roman choisie les aventures féeriques -, la nature des détails conservés, tout donne à penser que c'est le seul romanesque (caractéristique des remaniements) qui a séduit cet auteur. Cependant l'épopée n'est pas réellement absente ici, et même nous verrons que sa présence relève d'une démarche tout à fait volontaire.

D'emblée, le texte s'ouvre sur un rappel qui affirme fortement son inscription dans une tradition, par un résumé des aventures épiques d'Ogier:
Alors qu'Ogier soubz le roy Charlemaigne
Roy des François, a Empereur d'Espaigne
Eut mis a fin mainte guerre oultrageuse
Par sa proesse en ce victorieuse [...]

Vient ensuite une «complainte» d'Ogier menacé de naufrage, qui s'inspire fortement d'une prière du plus grand péril qui existait déjà dans la version en alexandrins ${ }^{8}$. Cependant, on ne saurait voir là une reprise probante de l'accent épique, qui au demeurant avait perdu de sa fermeté dans le roman. Curieusement, c'est alors dans un épisode entièrement inventé par le poète du XVI que nous trouvons le souvenir de l'épopée.

Dans le roman en prose, Ogier de retour en France suscitait de nombreuses jalousies et l'anneau de jeunesse, toujours menacé de vol, permettait de nombreux rebondissements. Habert s'inspire sans doute de ces péripéties pour écrire son troisième livre: Melchior, roi « de l'heur grand de son prince envieux » tente de voler l'anneau d'Ogier pendant son sommeil. Ne parvenant pas à s'en emparer, il s'enfuit avec la couronne du Danois. C'est alors que s'engage une véritable guerre, dont les images ne sont pas dénuées de grandeur. Ogier «faict sonner trompettes argentines »,

Lors eussiez veu venir de toute pars

Les chevaliers, qui fesoient leurs rampars,

Et en oyant des haultz fifres le son,

Devant Oger fesoient le limaçon.

L'armée se met alors en marche :

Ce conseil pris, se dressent estendards,

En ordre marchent hardys souldards.

Si qu'a les voir, et leur tumulte entendre,

Vous eussiez dict que la terre deust fendre.

Melchior entend «[...] les harnoys / Bruire et sonner pour Oger le Dannoys», puis le siège est mis au château du traitre:

Et en peu d'heure auprès de Chasteau Fort

Ont mis le cam affin que leur effort

\footnotetext{
${ }^{8}$ A cet égard, l'emploi que fait Habert du décasyllabe ne doit pas être interprété comme un retour au mètre proprement épique : c'est le mètre noble qu'il utilise quel que soit le genre qu'il aborde (avant de reconnaître la supériorité de l'alexandrin préconisé par les poètes de la Pléiade).
} 
Espouventast l'ennemye cohorte,

Voyant l'assault au plus pres de leur porte,

L'aer resonnoit du tumulte et grand bruict

Qui dedans l'ost du Dannoys court et bruict.

Melchior quant à lui fait appel à « deux empereurs de Barbarie [...] qui amenoient dessus la mer flottans, / A son secours cent mille combatans ".

Certes, la brièveté du texte empêche le déploiement complet des motifs épiques. Cependant, outre le topos thétorique du «or veissiez», on reconnait aisément l'évocation de la mêlée ( « vous eussiez veu voller bras, jambes et chef / Et corps navrez de lance en grand meschef»), du défi ("[Ogier] s'escrie, et haultement l'appelle: Viens Roy meschant, desloyal et rebelle [...] à nous deux le combat ») proposant un duel destiné à épargner des morts inutiles, l'ébauche même de ce duel où l'adversaire est jeté à bas de son cheval, la fuite des traitres et le siège (avec engins) qui naturellement dure sept ans.

Or, pour ce nouvel épisode, Habert a puisé son matériau dans l'ensemble de la Chevalerie Ogier. On reconnait ainsi dans ce «Chasteau Fort " « impossible de prendre» sur lequel Ogier bute sept ans le Castel-Fort où Ogier lui-même nargue Charlemagne, et le duel ébauché entre Ogier et Melchior reprend une semblable scène entre le Danois et son empereur. Quant aux rois venus en renfort de "Barbarie ", ils sont les dignes fils des chefs Sarrasins" toujours prêts à compléter les troupes des premiers assaillants.

Tout en manifestant constamment une grande liberté à l'égard du texte de référence, puisqu'il n'hésite pas ici à intervertir les rôles initiaux d'Ogier et de Charlemagne (c'est Ogier l'empereur en titre qui assiège Melchior appelé rebelle), Habert se soucie donc de conserver l'esprit épique, au point de le réinjecter dans son œuvre dans un épisode qui ne trouve pas sa justification dans la fidélité à sa matrice. Nous tenterons ultérieurement d'élucider la fonction de ce passage, mais auparavant il nous faut nous arrêter sur la conclusion de cette guerre: Ogier en effet ne parvient pas à faire tomber le château, et il lui faut attendre que son fils Hector, parvenu à l'âge adulte, le venge après avoir reçu l'adoubement (effectué selon les règles, marqué par «l'accolée»). Or Hector utilise la ruse : «contrefaisans l'estat de mercature», il entre sans encombre dans Château Fort, avant, la nuit venue, d'en ouvrir les portes pour ses compagnons. Encore une fois, on ne peut s'empêcher de reconnaitre un souvenir épique, cette fois La Prise d'Orange et Le Charroi de Nimes.

De fait, l'utilisation libre du roman d'Ogier, comme la référence à la geste de Guillaume $s$ 'inscrivent dans une pratique constante de notre texte. Habert multiplie en effet les échos de toute la littérature héroïque médiévale : sont évoqués Charlemagne, Renaut, Olivier, Roland mais aussi Arthur et la table ronde, et surtout Lancelot, notamment dans un passage directement inspiré de La Mort du Roi Arthur (\$ 51-52). Après son repas de noces, Ogier contemple sur le mur du palais de Morgue l'histoire gravée des «faictz de grand pris,/ Que pour Genevre [Lancelot] avoit entrepris »(premier livre). Plus loin, on pense encore au Lancelot en prose et au cimetière de la Douloureuse Garde lorsque la fée montre à Ogier les tombeaux des chevaliers de la table ronde, où Habert mêle Arthur, Lancelot, Gauvain, mais

\footnotetext{
${ }^{9}$ Ce n'est sans doute pas sa connotation orientale qui justifie le prénom du traitre Melchior : c'est le nom du traitre chambellan dans Théseus de Cologne, roman d'aventures tardif qui a également fait l'objet de nombreuses éditions au Xví siècle.
} 
aussi Perceforest, Giglan (le Guinglain du Bel Inconnu, lui-même un temps amant d'une fée), et Isaye le Triste (fils de Tristan et d'Iseut, héros d'un roman en prose du XIV siècle). Tous ces personnages sont bien connus au XVI siècle, témoins les nombreuse éditions imprimées dont nous disposons encore.

Le héros médiéval constitue donc la référence essentielle de l'œuvre, mais cette référence, loin d'être unique, est conçue comme un maillon d'une chaine ininterrompue de héros, qui trouve ses origines dans l'Antiquité. Ainsi, dès les premiers vers, c'est l'épopée grecque qui fournit le cadre des comparaisons servant à définir Ogier : «[...] le Dannoys a esté denigrand / le bruict d'Aiax d'Achilles et d'Hector ${ }^{10}$ » puis il est dit plus beau que Pâris (tandis qu'une nymphe lui présente une pomme d'or). Sur le mur qu'il contemple, les hauts faits des chevaliers jouxtent ceux d'Hector, de Pâris, d'Achille, mais également des Romains et d'Hannibal. Durant le banquet, la discussion roule sur Mars, Pallas, les «sept saiges de Grece», avant l'apparition d'Orphée et d'Amphion. Enfin, devant les tombeaux, c'est le Danois lui-même qui établit la perspective globale: il compare les chevaliers des légendes celtiques aux chevaliers de France, puis à Hector, César, Camille, avant de se situer luimême dans cette théorie de héros.

De prime abord, on croit reconnaître là une pratique esthétique courante à la Renaissance : au delà de la référence obligée à l'Antiquité, apparaît ce goût du mélange, de la varietas, qui, jusque dans la reprise affichée d'une œuvre médiévale, élargit le cadre à toute la littérature héroïque du Moyen Age pour finalement la dépasser et l'intégrer à un tableau exhaustif. De fait, à bien y regarder, l'esthétique du disparate, du désordre, n'est qu'une apparence ici : la comparaison menée par le personnage principal dit assez que ce n'est pas une pratique esthétique, mais bien une perspective politique qui gouveme la composition de notre texte. Comment en effet ne pas rapprocher cette généalogie héroïque des mythes de fondation de la monarchie française ? ${ }^{11}$

\section{Un miroir du prince}
Cest ceuvre la, Sire, sans point de faulte
Combien qu'il soit de fables tout rempli
Ha toutefois de verité le pli
En mains endroicts, car soubs la couverture
De fabuleuse, et menteuse escripture
Un sens ya soubs lequel on comprend
Toute prudence et bien vivre on apprend.

Cette épître ${ }^{12}$, qui ouvrait les Métamorphoses d'Ovide traduites par F. Habert pour Henri II, vaut également pour notre texte: la fable doit être prétexte à une leçon destinée au souverain. Car Habert apporte une modification frappante à son modèle: dans le roman en prose,

\footnotetext{
${ }^{10}$ Ogier donnera le prénom d'Hector à son fils en hommage explicite au héros troyen :

Oger ung tel nom luy octroye,

Pour le renom du preux Hector de Troye.

Dans le roman en prose, l'enfant est Meurvin.

${ }^{11}$ On pense bien sûr à la Franciade de Ronsard, qui fera descendre Francus d'Hector.

${ }^{12}$ Reproduite par H. Franchet, op. citée, p XXXVIII.
} 
Ogier " époux » de Morgue n'assume pas à proprement parler le pouvoir : seul Arthur, bien présent, reçoit ce nom à Avalon. Or chez F. Habert, Arthur a disparu, et Ogier devient solennellement roi dès ses noces.

De même, sur un plan formel, il n'est que de considérer les transformations apportées à la structure de l'œuvre de départ pour saisir que le but poursuivi est bien l'édification du Prince. Habert a en effet divisé en trois parties une matière à l'origine étendue sur plusieurs chapitres, en intégrant la trame narrative du texte-source à un plan d'ensemble qui lui confère un nouveau sens.

Ainsi, le premier livre se définit comme le temps de l'initiation. A travers les combats inspirés ${ }^{13}$ du roman, nous avons vu qu'Ogier gagne son mariage, puis surtout son droit à régner. Logiquement, le second livre est alors entièrement consacré à l'exercice du pouvoir. Après avoir fait s'exercer ses chevaliers « jour en jour », Ogier les convoque pour édicter ses lois en un fort long discours, puis expose, lors d'un banquet, son idéal de gouvernement. Enfin, par crainte de sombrer dans l'oisiveté, il entreprend de faire décorer les tombeaux des grands héros, ce qui fournit l'occasion d'une nouvelle réflexion sur la postérité.

Le troisième livre est le livre d'Hector, fils d'Ogier et de Morgue, qui vengera son père de la trahison de Melchior. L'intégration de cette troisième partie (pourtant consacrée à un nouveau personnage) à la réflexion sur le pouvoir ne fait aucun doute ${ }^{14}$. Les aventures d'Hector posent le problème, essentiel pour Habert ${ }^{15}$, des successeurs du roi. Le troisième livre applique donc le programme édicté par Ogier au cours du banquet du deuxième: les successeurs

Ne doibvent point par leurs predecesseurs

Avoir louange, ains qu'il fault de luy-mesme

Chercher honneur, coronne, et diaderne.

Au demeurant, ce troisième livre offre l'avantage de permettre la mise en scène de l'éducation du jeune prince, élevé « Comme Romains qui avecque l'art bellicque / Scavoient la lettre et fleurs de rhetoricque. » afin de conjuguer savoir, «corps vestu de grand povoir » et "louable vertu».

Car les idées avancées ne brillent pas par leur originalité : Habert s'inscrit honnêtement dans la pensée de son temps et de la tradition monarchique française. A travers Ogier, il peint un roi qui doit être un guerrier éprouvé (les tournois lui évitent l'oisiveté physique) et un sage qui fait régner la concorde sous l'égide des lois chrétiennes ${ }^{16}$.

\footnotetext{
${ }^{13}$ Notons cependant que la valeur symbolique de la seconde épreuve (inventée par Habert) est fortement appuyée : avant de rencontrer le serpent-dragon, Ogier en un « lieu d'obscurité [qui] sembloit estre ung enfer " doit vaincre vingt-deux géants armés de flèches venimeuses. L'évocation de l'enfer fait écho à celle du paradis, et marque bien l'importance de l'imaginaire chrétien chez notre auteur.

${ }_{14}^{14}$ Rappelons que Melchior, venu chercher l'anneau, s'empare finalement de la couronne d'Ogier, c'està-dire du symbole de sa fonction.

${ }^{15}$ Un chapitre complet du Philosophe Parfait est consacré à ce thème : « La louange des prédécesseurs ne vient aux ignobles successeurs » (voir surtout les v. 295 à 300, ed. citée, p 18). C'est d'ailleurs un des lieux communs de la philosophie humaniste que ce problème de la conservation de la noblesse.

${ }^{16}$ Pour une fois, Habert martèle son propos (se faisant sans doute ici le relais de Henri II) : le peuple doit vivre "soubz une loy, soubz ung bien, soubz ung prince". On reconnait la concentration des pouvoirs entre les mains du roi, qui s'affirme au Xvi' siècle.
} 
Ogier est en effet un roi générateur d'une norme, un roi législateur. Le terme même de loi, relayé par celui, plus concret, d'édit, constitue d'ailleurs un véritable réseau structurant ces discours consacrés au pouvoir, l'antithèse étant représentée par la transgression (" obéissance " et "ordonnance » s'opposant d'emblée aux " transgresseurs") incamée dans le troisième livre en Melchior le «desloyal» (l'épithète le caractérise presque systématiquement), « de la loy chestienne transgresseur» (il s'agit donc bien de faire du roi le garant de l'ordo mundi divin). Melchior est d'ailleurs jugé par le Sénat selon la loi, même si «le Dannoys (...) [le] fait mourir par les loix qu'il a faictes " mais auxquelles il se plie lui-même : un roi doit observer

La by qu'il a faicte, sans reserver

Chose qui peult luy donner liberté

Pour ses subjectz mettre en captivité.

Peignant un roi courtois et libéral, Habert offre enfin dans le banquet un exemple de débat où chacun prend la parole, mais où en dernier recours c'est Ogier

Qui contenta toute sa seigneurie

De son parler doux, et scientificque.

Clémence et Savoir font bien d'Ogier un roi humaniste sur le modèle de Gargantua.

Plus originale est l'exploration du rôle tenu par les arts, et surtout celui du poète, auprès du prince. On se souvient que dans le roman en prose, Ogier était accueilli à Avalon par un ballet de fées: Habert a manifestement pris plaisir à développer ce passage qui lui offre l'occasion de mettre en scène un gracieux orchestre (il nomme Avalon le « lieu mélodieux " dans le second livre) et il évoque également l'art du peintre à plusieurs reprises. Cependant les banquets permettent surtout de dresser une liste des multiples poèmes, véritable feux d'artifice des genres (se mêlent dizains, rondeaux, virelais, balades ${ }^{17}$ et épitres), chantés par les fées, Orphée ou Amphion ${ }^{18}$, et tous composés à la gloire d'Ogier, certains par Morgue
Pour collauder les dictz, armes, et faictz
De son amy, accomplis et parfaictz,
Le tout au long dedans le mur gravé,
Sans que le temps eut l'escript depravé.

Comment ne pas lire ici la présence de l'auteur au cœur de son œuvre, et l'écho des idéaux d'un poète officiel, dans ces louanges devenues immortelles?

La poésie à la cour doit chanter le souverain, mais l'art n'est pas l'affaire de celui qui gouverne: Ogier désireux de faire décorer les tombeaux des grands chevaliers trouve l'œuvre déjà accomplie par Praxitèle, et surtout il enseigne à son fils que «le scavoir evangelicque » est «plus precieux que n'est l'art poeticque » (troisième livre).

\footnotetext{
${ }^{17}$ Habert n'appartient pas à la nouvelle école littéraire : ces genres, décriés par Du Bellay dans Défense et Illustration de la Langue Francaise, sont ceux des Grands Rhétoriqueurs.

${ }^{18}$ La présence d'Amphion n'est peut-être pas gratuite : c'est lui qui construisit les murailles de Thèbes par le seul pouvoir de sa lyre. Cependant la convocation de cette figure est également un lieu commun à la Renaissance.
} 
Car Habert n'oublie pas la fonction des Visions. Traditionnellement perçu comme un " défenseur de la morale à la cour ", il fait également porter sa leçon sur l'affirmation de la loi chrétienne. Ogier roi exige le respect de la charité, le refus de la simonie, de l'hérésie et de l'hypocrisie.

Cette forte affirmation du respect de la religion justifie dès lors le texte de Habert, puisque s'il se livre à cet " art poétique " mineur, c'est pour diffuser le " savoir évangélique » essentiel. Elle permet sans doute également de faire accepter d'autres aspects de l'ouvrage: cette édification du prince est en effet dédièe à une femme ${ }^{19}$, pour laquelle l'auteur définit ses Visions comme une «leçon [...] où [elle] pourr[a] prendre quelque plaisir " ${ }^{20}$. C'est peutêtre donc cette dédicace féminine qui explique, outre les passages traditionnellement destinés à distraire la cour (ainsi les louanges du cuisinier Patinus, inventé sur patina, sorte de plat creux, mais aussi la peinture des fêtes et des tournois), les développements sur l'amour, généralement placés dans la bouche de Morgue. En effet, l'amour offert à Ogier par la fée ( J'entens amour par mariaige ferme / Et qui deux cueurs en ung vouloir enferme » premier livre) permet d'établir une distinction nette entre cupido (défini comme un poison) et l'amour « loyalle et ferme » qu'elle glorifie dans un long exposé.

Edification complète, donc, dans ces Visions, truffées de discours qui condensent une morale politique, religieuse, amoureuse même, en même temps qu'elles donnent à voir, qu'elle mettent en scène un modèle de vie à la cour suivant une pensée généralement humaniste, et parfaitement adaptée au public cultivé qui entourait Henri II.

\section{Conjonction du matériau médiéval et de la visée « humaniste»}

Reste à faire coïncider l'habillage médiéval et le contenu "Renaissance " dans un texte qui, rappelons-le, est unique en son genre dans l'œuvre de François Habert, généralement plus inspiré par la mythologie: qu'a donc à voir avec l'édification du Prince la légende d'Ogier le Danois?

Il n'est pas inutile de rappeler ce qu'est un poète officiel : sa fonction est de chanter Dieu et le Roi, c'est-à-dire qu'il assume un rôle politique, non seulement en se faisant vecteur d'une idéologie religieuse et royale, mais également en proposant un idéal entraînant aussi bien le souverain que l'ensemble de la collectivité.

Dans ce contexte, on comprend aisément l'intérêt que pouvait représenter la reprise des aventures d'un héros d'épopée, puisque ce genre répond exactement aux exigences de l'art épidictique. Or, dès le début de l'ouvrage, l'auteur rappelle clairement qu'Ogier est avant tout un héros épique, et la brièveté des allusions faites aux exploits antérieurs du Danois, (de même que l'ouverture in medias res) donne à penser que F. Habert s'adresse à un public qui connaît bien ceux-ci. Doté d'un passé glorieux, Ogier présente surtout l'avantage de devenir roi, un roi guerrier qui justifie son pouvoir les armes à la main : on reconnaît ici l'image du prince-héros qui traduit bien au XVI ${ }^{\mathrm{e}}$ siècle les aspirations de la collectivité. Nous reviendrons sur la signification que revêt la guerre contre Melchior, mais il faut se souvenir que F. Habert

\footnotetext{
${ }^{19}$ Madame la duchesse de Touteville. Le Philosophe Parfait était déjà dédié à Monseigneur François de Bourbon, duc de Touteville, et à Madame Andrienne de Touteville. Le nom de cette famille indique assez sa proximité avec le pouvoir.

${ }^{20}$ Prodesse et delectare : rappelons que F. Habert est traducteur d'Horace.
} 
a ajouté cet épisode d'un conflit de type féodal ${ }^{21}$ en s'appliquant à reproduire les formules et un peu de la poésie guerrière des chansons de geste, propres à susciter l'émotion d'un roi aussi belliqueux qu'Henri II.

On sait cependant que l'épopée à la Renaissance est en crise et se cherche une nouvelle définition : ainsi Peletier prône-t-il l'emprunt aux romans des « aventures des chevaliers, les amours, les voyages, les enchantements (...) $)^{22}$. De façon générale, le discours moral de l'époque se déploie volontiers dans un cadre romanesque et merveilleux (au moment où Habert écrit, on commence à lire en France le Roland Amoureux et Roland Furieux), le recours à l'imaginaire charmant en même temps qu'il suscite l'intérêt et favorise la persuasion. Sans pouvoir affirmer que $\mathrm{F}$. Habert connaissait les textes italiens, on peut cependant penser qu'il était sensible à cet " air du temps ». Or, tous les remaniements tardifs d'épopée offrent un grand nombre d'aventures qui mêlent différents types de merveilleux. Mais si plusieurs héros rencontrent Morgue ou visitent Avalon, Ogier est le seul qui règne durablement sur Féerie et c'est précisément ce passage qui suscite l'intérêt de F. Habert.

Enfin, la structure même de l'épisode initial n'est pas sans rapport avec celle des Visiones chrétiennes: Ogier croit sa demière heure venue lorsqu'il aborde à l'île merveilleuse (au statut ambigu: elle cache, dès le roman en alexandrins, ce qui ressemble fort au jardin d'Eden). Son séjour en Féerie représente une mort au monde, et son retour en France après deux cents ans est perçu comme une résurrection. Seule la thématique de la conversion (ou du moins du repentir) est absente, mais c'est parce qu'Ogier, héros épique, se définit $a$ priori comme champion de la foi. On verra comment Habert a su habilement exploiter ce fonds chrétien selon ses propres objectifs.

La conjonction de ces éléments propres au modèle, à savoir un pieux héros d'épopée amené à régner sur le royaume de Morgue, offrait à $F$. Habert un matériau unique, qui permettait de coordonner portée religieuse et réflexion sur le pouvoir dans un cadre élevé. En effet, les Visiones ont assurément une valeur moralisante, et véhiculent les idéaux chrétiens, mais elles n'occasionnent jamais une mise en scène de l'exercice du pouvoir royal: quel personnage pourrait être mis en situation de régner sur l'enfer ou le paradis?

Or le séjour d'Ogier en Féerie, en puissance conçu (dès le roman) comme l'histoire de l'établissement d'un règne, depuis la prime accession au pouvoir jusqu'à l'affermissement dans une descendance, permet d'abord de développer un miroir du prince valorisé par le fait qu'il s'exerce dans un univers merveilleux supérieur. Rappelons que dans le cadre même de l'épopée médiévale, la confrontation des héros de chanson de geste et de certains personnages arthuriens représente une promotion pour le héros épique: l'amour de la fée Morgue rehausse la valeur d'un chevalier reconnu jusque dans l'Autre Monde ${ }^{23}$. Cette idée n'a pas

\footnotetext{
${ }^{21}$ A cet égard, il faut voir dans cet épisode une allusion à la maitrise toujours difficile de la grande noblesse, à une époque où le roi doit encore achever la légitimation de son pouvoir (certes Melchior est un roi, mais c'est avant tout un sujet de son Prince). Il nous paraît significatif que F. Habert n'ait pas réservé le récit de la guerre à la lutte d'Ogier contre les Luytons, pourtant très développée dans l'œeuvre originelle : les Luytons représentent une menace extérieure, et sont au demeurant trop marqués par leur nature féerique. Il ne s'agit plus de mettre en scène l'étrangeté du Sarrasin : Melchior est un homme semblable à Ogier.

${ }^{22}$ L'Art Poétique, cité par D. Madelénat in L'Epopée, Paris, PUF, 1986, p. 218.

${ }^{23}$ Sur ce sujet, voir F. Suard, "La Bataille Loquifer et la pratique de l'intertextualité au début du XII" siècle ", Chanson de geste et tradition épique en France au Moyen Age, Caen,Paradigme, 1994.
} 
échappé à Habert: Morgue explique chez lui son amour pour Ogier par un discours explicitement comparatif :

Je t'ay receu comme le plus notable,

Des chevaliers au bellicqueux couraige [...]

Ce qui a faict mon ceil n'estre endormy,

Entre plusieurs te choisir pour amy. (fin du deuxième livre).

En effet, l'accueil d'un héros épique dans l'Autre Monde justifie également la convocation de l'ensemble des héros médiévaux, et par-delà le Moyen Age, l'établissement d'une lignée hérö̈que qui prend sa source dans l'Antiquité. Cette mise en perspective générale contribue finalement à l'élaboration d'un «miroir superlatif » du prince renforcé par un effet de mise en abyme : c'est au centre de l'ouvrage qu'Ogier se compare aux grands héros avant que Morgue ne lui accorde l'avantage, éliminant ainsi tout autre objet de comparaison pour le lecteur royal qu'Ogier lui-même.

D'autre part, nous avons rappelé qu'Ogier en tant que héros d'épopée est par essence défini par sa foi, et que le récit de son séjour en Féerie offre des similitudes avec le genre des Visions. Dès lors, l'épisode pouvait également devenir vecteur de morale chrétienne (le discours religieux d'Ogier se trouvant cautionné par son statut de héros épique), au prix d'une légère torsion du modèle: dans le roman en prose, Morgue et les fées sont les servantes de Dieu, alors que dans le texte de F. Habert, les habitants de l'île se convertissent à la religion chrétienne sous l'impulsion de leur nouveau roi.

Sans doute faut-il accorder une valeur politique à cette transformation: Ogier exigeant de son peuple l'abandon de l'« hérésie » se fait l'écho de Henri II en lutte contre la Réforme. Cependant l'intérêt du texte réside également dans une nouvelle exploitation du merveilleux: dans le même temps que la mise en scène de l'île utopique «égaie " le discours moral et cautionne l'image d'un règne idéal, la féerie se dissout au profit de l'affirmation d'une orthodoxie chrétienne. A plusieurs reprises, il apparait en effet clairement que la fonction d'Ogier devenu roi est de détruire les «normes » féeriques pour leur substituer la loi de Dieu (on se souvient qu'Ogier est un roi « législateur»).

Deux épisodes du roman d'Ogier sont ainsi revus par Habert : d'une part, celui de la naissance du héros. Dans le texte-source, les fées présidaient à cette naissance et émettaient des prophéties. Dans l'œuvre du Xvie siècle, Ogier lui-même interdit aux trois fées

Qui employer vouloient labeur et cure,

Pour de l'enfant destiner l'adventure

d'exercer leur pouvoir: «[...] ung seul dieu, de l'humaine nature / Pere et autheur, cognoist chose future. ». La thématique (ensuite devenue lieu commun) de la prédestination par les fées n'est ici convoquée que pour mieux être révoquée.

Comme la naissance, la mort réintègre l'ordre chrétien: dans la tradition, la mort d'Ogier est liée à la consomption d'un flambeau et à l'enlèvement par Morgue. Chez Habert, lorsque la fée évoque cette fin couronnée par l'accueil de Jupiter « au celeste habitacle », Ogier réagit en chrétien: 
Oyant ces motz, helas il ne pensoit

Sinon en ce qu'ung fidele concoit. (fin du deuxième livre).

Aspirant au ciel, il décide aussitôt d'une lecture journalière de la Bible ( la loy evangelicque »).

Sans doute faut-il également rattacher à cette propagande chrétienne la mise à mal de deux clichés littéraires pourtant toujours exploités au XVI siècle : la roue de Fortune (qui selon Ogier «n'est rien sinon que fable poeticque ", puisque Dieu seul décide de notre sort) ; et Cupidon, avantageusement remplacé par la « loyalle amour » sanctifiée par le mariage. A cet égard, il est intéressant de constater que dans les deux cas, Habert adopte une position de moraliste chrétien qui s'oppose aux poètes : ainsi à propos de l'amour, il se livre à une critique des peintures traditionnelles de ce demier. Chez Habert, l'art est toujours inférieur, inféodé, à la visée morale.

Ce mépris affiché pour les lieux communs merveilleux doit aussi être mis en relation avec la portée politique du texte. En effet, pourquoi Ogier, ce roi modèle, ne peut-il venir à bout du rebelle Melchior? Ce fait doit probablement être rapproché d'une particularité du récit de la guerre entre les deux hommes. Avant la bataille, Habert prête à Melchior un long discours (soixante-quatre vers) où il justifie sa révolte par une remise en cause de la légitimité du pouvoir d'Ogier:

De tout ce regne il est coronné roy,

Non toutesfois qu'il fust si florissant,

Sans la vertu d'ung bel anneau puissant,

Dont Morgue la haultement estrené

[...]

Mais quand il est de l'anneau devestu,

Certainement il n'a plus grande force

Que boys pourry, ou une seiche escorce.

La harangue de Melchior permet à Habert de se dissocier de son personnage principal, afin d'en montrer les limites. La faute d'Ogier est peut-être de fonder sa force sur la magie: finalement seul son fils Hector, qui ne fait confiance qu'à sa ruse (sur l'exemple plus " pur ", du point de vue des genres, de Guillaume), vaincra Melchior. Ainsi le merveilleux est-il révoqué jusque dans le héros-modèle, ce qui ménage la possibilité pour le lecteur-roi d'une prise de distance valorisante.

On voit bien ici comment l'imaginaire féerique est manipulé par Habert: un peu à la façon dont le héros épique se trouvait promu par son entrée dans l'Autre Monde, la religion se trouve apparemment rehaussée par sa reconnaissance dans ce monde utopique présenté comme une référence. Mais en réalité, faire triompher la norme chrétienne dans l'univers féerique équivaut à détruire celui-ci, au profit de celle-là. Le merveilleux n'est plus qu'un décor en trompe l'œil: le mieux qu'il puisse lui arriver est d'obéir aux mêmes règles que le monde réel, ou du moins de sa projection idéalisée. Au total, il s'agit donc bien de célébrer le triomphe de l'idéologie religieuse que sert Habert. 


\section{Conchusion}

Il ne saurait être question d'accorder aux Visions d'Oger une importance qu'elles n'ont pas dans l'histoire littéraire de la Renaissance. La qualité inégale d'un texte au demeurant relativement bref montre assez que F. Habert n'est ni Ronsard ni Du Bellay.

Cependant cet échantilion de littérature " officielle » nous fournit matière à réflexion sur l'évolution du genre épique au XVI siècle. Habert n'a pas la prétention d'écrire l'épopée que son statut exigerait, et qu'aucun de ses contemporains ne parviendra d'ailleurs à offrir à son temps. Néanmoins, tel qu'il est, ce texte témoigne de certaines des forces qui travaillent le genre : victoire du romanesque, développement du didactisme, mais également tiraillements entre un merveilleux féerique et une religion triomphante (résolu chez d'autres auteurs par l'allégorie, et chez Habert par la soumission de l'un à l'autre).

Dans le même temps, cette œuvre montre que l'épopée manifeste encore une certaine vitalité : d'abord parce que, même sous une forme abâtardie (ici le roman d'Ogier en prose), la chanson de geste est toujours lue (et à l'évidence bien connue) à la cour.

Ensuite et surtout, cette réécriture prouve bien que le $\mathrm{XVI}^{\mathrm{e}}$ siècle sait exactement à quoi sert l'épopée. Le recours à un héros épique, l'arrière-plan constitué par ses aventures, viennent étayer le déploiement d'une propagande théologique et politique qui forment le cour même de la problématique du genre : il s'agit toujours de souder une communauté, ici autour d'un Prince-héros qui enseigne les valeurs chrétiennes. Tout l'intérêt du texte réside alors dans ce qu'il nous révèle des nouvelles données politiques et religieuses. Plus de luttes aux frontières du royaume (l'ennemi est d'abord à l'intérieur); l'affirmation écrasante d'une monarchie autoritaire qui n'éprouve plus même le besoin de s'appuyer sur le bras armé d'un héros autre que le souverain; et le triomphe d'une religion devenue loi. Fort heureusement, ce message ô combien sérieux est pour nous tempéré par l'évocation discrète de la naissance d'un art de vivre qui, malgré tout, fait de notre poète officiel un lointain cousin des thélémites, et à coup sûr un admirateur des gracieuses nymphes qui danseront bientôt sous le ciseau de Jean Goujon.

Emmanuelle Hoyer-Poulain

(Université d'Arras) 